\title{
Highly expressed host genes in the cells of monkeys infected with SARS-Cov2 - eukaryotic translation elongation factors, cathepsin L, galectin, vimentin
}

Sandeep Chakraborty

\section{Letter}

A recent pre-print has used Nanopore sequencing to 'describe aspects of coronavirus evolutionary genetics revealed from these data, and provide the first direct RNA sequence of SARS-CoV-2, detailing coronaviral subgenome-length mRNA architecture' [1]. They have analyzed reads matching to the SARS-Cov2. Here (Table 1) I analyze reads matching to the host cell (ignoring ribosomal genes) - the full list is in SI:hostgeneexpression.txt.

\section{Eukaryotic translation elongation factors}

This review details how 'viral pathogens usurp these abundant cellular proteins for their replication' [2].

\section{S100 calcium binding proteins}

These proteins seem to be host defence mechanisms as in this study 'S100A1, S100A2 and S100A6 in sO cells resulted in the efficient inhibition of HCV replication' [3].

\section{Cathepsin L}

This - and not TMPRSS2 [4] - seems to be the protease involved in SARS-Cov2 [5]. Note, Cathepsin L 'functionally cleaves the severe acute respiratory syndrome coronavirus class I fusion protein upstream of rather than adjacent to the fusion peptide' [6] and its inhibition prevented virus entry' [7]. There is also a contrarian view since human coronavirus NL63 does not use Cathepsin L to infect ACE2-expressing cells [8].

\section{Galectin}

Galectins have a role in virus infection [9]. In fact this is a "viral evolution strategy in which coronaviruses stole a galectin from hosts, incorporated it into their spike protein, and evolved it into viral receptor-binding domains with altered sugar specificity in contemporary $\mathrm{BCoV}$ or novel protein specificity in contemporary MHV' [10].

\section{Vimentin}

'Surface vimentin is critical for the cell entry of SARS-CoV' [11].

\section{References}

1. Taiaroa G, Rawlinson D, Featherstone L, Pitt M, Caly L, et al. (2020) Direct RNA sequencing and early evolution of SARS-Cov-2. bioRxiv . 
2. Li D, Wei T, Abbott CM, Harrich D (2013) The unexpected roles of eukaryotic translation elongation factors in RNA virus replication and pathogenesis. Microbiol Mol Biol Rev 77: 253-266.

3. Tani J, Shimamoto S, Mori K, Kato N, Moriishi K, et al. (2013) Ca2+/S100 proteins regulate HCV virus ns $5 \mathrm{a}-\mathrm{fkbp} 8 / \mathrm{fkbp} 38$ interaction and HCV virus RNA replication. Liver International 33: $1008-1018$.

4. Hoffmann M, Kleine-Weber H, Schroeder S, Krüger N, Herrler T, et al. (2020) SARS-Cov-2 cell entry depends on ACE2 and TMPRSS2 and is blocked by a clinically proven protease inhibitor. Cell .

5. Mori Y, Yamashita T, Tanaka Y, Tsuda Y, Abe T, et al. (2007) Processing of capsid protein by cathepsin L plays a crucial role in replication of Japanese encephalitis virus in neural and macrophage cells. Journal of virology 81: 8477-8487.

Table 1: Highly expressed host genes in the cells of monkeys infected with SARS-Cov2: Sequencing data obtained from Accid:PRJNA608224 [1]. Excluded ribosomal and mitochondrial genes.

\begin{tabular}{|c|c|}
\hline Read counts & Description (Human accession id) \\
\hline 2442 & NM_001402.6 eukaryotic translation elongation factor 1 alpha 1 (EEF1A1), mRNA \\
\hline 1519 & XM_017002033.1 S100 calcium binding protein A6 (S100A6), \\
\hline 1309 & NM_006098.4 receptor for activated C kinase 1 (RACK1), mRNA \\
\hline 1189 & XM_011516549.3 VGF nerve growth factor inducible (VGF), \\
\hline 1134 & NM_021103.4 thymosin beta 10 (TMSB10), mRNA \\
\hline 1070 & XM_024446695.1 aldo-keto reductase family 1 member B (AKR1B1), \\
\hline 1063 & XM_017014293.2 cathepsin L (CTSL), \\
\hline 1025 & NM_002305.4 galectin 1 (LGALS1), mRNA \\
\hline 1024 & NM_006082.3 tubulin alpha 1b (TUBA1B), mRNA \\
\hline 970 & NM_021109.4 thymosin beta 4 X-linked (TMSB4X), mRNA \\
\hline 898 & NM_021009.7 ubiquitin C (UBC), mRNA \\
\hline 782 & NM_003295.3 tumor protein, translationally-controlled 1 (TPT1), \\
\hline 735 & XM_017012224.2 aldo-keto reductase family 1 member B15 (AKR1B15), \\
\hline 710 & NM_002520.6 nucleophosmin 1 (NPM1), \\
\hline 676 & XM_011511522.2 prothymosin alpha (PTMA), \\
\hline 670 & XM_006717500.2 vimentin (VIM), \\
\hline 643 & NM_001909.5 cathepsin D (CTSD), mRNA \\
\hline 642 & XM_017022313.2 pyruvate kinase M1/2 (PKM), \\
\hline 632 & NM_002415.2 macrophage migration inhibitory factor (MIF), mRNA \\
\hline 628 & NM_003329.4 thioredoxin (TXN), \\
\hline 626 & NM_001353346.2 enolase 1 (ENO1), \\
\hline 608 & XM_011542798.1 heat shock protein family A (Hsp70) member 8 (HSPA8), \\
\hline 595 & NM_001404.5 eukaryotic translation elongation factor 1 gamma (EEF1G), mRNA \\
\hline 564 & NM_001145199.1 chromosome 12 open reading frame 75 (C12orf75), mRNA \\
\hline 553 & XM_017024761.1 profilin 1 (PFN1), \\
\hline 551 & NM_001961.4 eukaryotic translation elongation factor 2 (EEF2), mRNA \\
\hline 543 & NM_178014.4 tubulin beta class I (TUBB), \\
\hline 509 & XM_024450193.1 aldolase, fructose-bisphosphate A (ALDOA), \\
\hline 494 & XM_017021370.1 serpin family A member 1 (SERPINA1), \\
\hline 485 & XM_024449908.1 annexin A2 (ANXA2), \\
\hline 479 & NM_016139.4 coiled-coil-helix-coiled-coil-helix domain containing 2 (CHCHD2), \\
\hline 457 & NM_002032.3 ferritin heavy chain 1 (FTH1), mRNA \\
\hline
\end{tabular}


6. Bosch BJ, Bartelink W, Rottier PJ (2008) Cathepsin L functionally cleaves the severe acute respiratory syndrome coronavirus class I fusion protein upstream of rather than adjacent to the fusion peptide. Journal of virology 82: 8887-8890.

7. Simmons G, Gosalia DN, Rennekamp AJ, Reeves JD, Diamond SL, et al. (2005) Inhibitors of cathepsin $\mathrm{L}$ prevent severe acute respiratory syndrome coronavirus entry. Proceedings of the National Academy of Sciences 102: 11876-11881.

8. Huang IC, Bosch BJ, Li F, Li W, Lee KH, et al. (2006) SARS coronavirus, but not human coronavirus NL63, utilizes cathepsin 1 to infect ACE2-expressing cells. Journal of Biological Chemistry 281: 31983203.

9. Wang WH, Lin CY, Chang MR, Urbina AN, Assavalapsakul W, et al. (2019) The role of galectins in virus infection-a systemic literature review. Journal of Microbiology, Immunology and Infection .

10. Peng G, Xu L, Lin YL, Chen L, Pasquarella JR, et al. (2012) Crystal structure of bovine coronavirus spike protein lectin domain. Journal of Biological Chemistry 287: 41931-41938.

11. Yu YTC, Chien SC, Chen IY, Lai CT, Tsay YG, et al. (2016) Surface vimentin is critical for the cell entry of SARS-Cov. Journal of biomedical science 23: 14. 